\title{
Exponential decay of solutions to nonlinear elliptic equations with potentials
}

Reika Fukuizumi and Tohru Ozawa

Dedicated to Professor Jun Uchiyama on the occasion of his sixtieth birthday

\begin{abstract}
Exponential decay estimates are obtained for complex-valued solutions to nonlinear elliptic equations in $\mathbb{R}^{n}$, where the linear term is given by Schrödinger operators $H=-\Delta+V$ with nonnegative potentials $V$ and the nonlinear term is given by a single power with subcritical Sobolev exponent in the attractive case. We describe specific rates of decay in terms of $V$, some of which are shown to be optimal. Moreover, our estimates provide a unified understanding of two distinct cases in the available literature, namely, the vanishing potential case $V=0$ and the harmonic potential case $V(x)=|x|^{2}$.
\end{abstract}

Mathematics Subject Classification (2000). 35J60, 35B40.

Keywords. Exponential decay, nonlinear elliptic equations, bound states

\section{Introduction}

In this paper we study the asymptotic behavior at infinity of solutions to the elliptic equations of the form

$$
-\Delta u+V u+\omega u=|u|^{p-1} u,
$$

where $u$ is a complex-valued function on $\mathbb{R}^{n}, V$ is a given nonnegative function on $\mathbb{R}^{n}, \Delta$ is the Laplacian in $\mathbb{R}^{n}, \omega \in \mathbb{R}, p>1$, and $n \geq 1$. The purpose in this paper is to present exponential decay of solutions to (1.1) with specific rate given in terms of the linear potential $V$. We describe the exponential decay by means of $L^{2}$ and $H^{1}$ estimates, local $L^{2}$ estimates, and pointwise estimates. In the special case where $V(x)=|x|^{\theta}$ with $\theta>0$, the rate of exponential decay is shown to be optimal by lower bound estimates due to Uchiyama [36, 37]. Moreover, the rate reduces to the two well-known results of the kind, namely, the vanishing potential case $V=0$ [3] and the harmonic potential case $V(x)=|x|^{2}[11,12,15]$, so that it gives a unified understanding how potentials come into play in the phenomena of exponential decay of solutions. We note here that the method in [3] for $V=0$ 
is applicable to complex-valued solutions, while the resulting exponential decay is of order $O\left(e^{-C|x|}\right)$ which is not always optimal. It has been known only by ODE methods and the maximal principle in $[11,12,15]$ that Gaussian decay $O\left(e^{-C|x|^{2}}\right)$ is proved for positive, radial solutions to (1.1) with $V(x)=|x|^{2}$. Equations of the form (1.1) with $V=0$ have been extensively studied (see for instance [2, 19, 20, 21, $33]$ ), while there arises a new interest in (1.1) with $V(x)=|x|^{2}[11,12,14,15,26]$. Equations of the form (1.1) are of special interest in connection with standing waves for nonlinear Schrödinger equations in the attractive case

$$
i \partial_{t} v+\Delta v-V v=-|v|^{p-1} v
$$

where $v$ is a complex-valued function in space-time $\mathbb{R}^{n+1}$. We call $v$ a standing wave for (1.2) if $v(t, x)=e^{i \omega t} u(x)$ and $u$ solves (1.1). We refer the reader to $[3,13,22]$ for general studies on the Cauchy problem for (1.2) and to [1, 4, 9, 30, $31,38,39]$ (when $V=0$ ), [5, 6, 7, 8, 9, 16, 23, 27, 40, 41] (when $V \neq 0$ ) for stability and instability results on standing waves for (1.1).

To state our main results precisely, we introduce notation and conventions. We consider solutions of (1.1) in the space $\Sigma \equiv\left\{u \in H^{1} ; V^{1 / 2} u \in L^{2}\right\}$ equipped with scalar product

$$
(u, v)_{\Sigma}=\int \nabla u \cdot \nabla \bar{v}+\int u \bar{v}+\int V u \bar{v}
$$

We denote by $2^{*}$ the Sobolev critical exponent defined as $2^{*}=2 n /(n-2)$ for $n \geq 3$ and $2^{*}=\infty$ for $n \leq 2$. For $p$ with $1<p<2^{*}-1$ solutions in $\Sigma$ make sense if both sides of (1.1) are considered in the dual space $\Sigma^{\prime} . \Sigma$ is a natural space where functionals such as

$$
\begin{aligned}
E(u) & =\frac{1}{2} \int\left(|\nabla u|^{2}+V|u|^{2}\right)-\frac{1}{p+1} \int|u|^{p+1} \\
S(u) & =\frac{1}{2} \int\left(|\nabla u|^{2}+V|u|^{2}\right)-\frac{1}{p+1} \int|u|^{p+1}+\frac{\omega}{2} \int|u|^{2}
\end{aligned}
$$

are well-defined. The above functionals play an important role in variational methods for (1.1), especially for the existence of solutions of (1.1). We refer the reader to $[2,5,15,20,21,26,34]$ for instance and do not go into details for the existence here.

We regard the elliptic differential operator $-\Delta+V$ in (1.1) as a Schrödinger operator $H=-\Delta+V$ in $L^{2}$ and, more generally, in $L^{q}$ with $1<q<\infty$ and as a bounded operator from $\Sigma$ to $\Sigma^{\prime}$, etc. We use the same notation $H=-\Delta+V$ indiscriminately when this causes no confusion. Here the Schrödinger operator $H=-\Delta+V$ in $L^{q}$ is understood to be the operator sum with domain $D(H)=$ $\left\{u \in W_{q}^{2} ; V u \in L^{q}\right\}$ and $V$ to be the multiplication operator by a nonnegative function $V \in L_{l o c}^{q}$. We refer to $[24,25,29]$ for $L^{q}$ theories for the Schrödinger operators.

We now introduce the main assumptions on $V$. Throughout the paper we fix $\varepsilon$ as an arbitrarily small constant with $0<\varepsilon<1$. 
(V1) For any $q$ with $1<q<\infty, H$ forms a maximal accretive operator in $L^{q}$.

(V1) There exists $q$ with $n<q<\infty$ such that distributional derivatives $\partial_{j} V, 1 \leq$ $j \leq n$, satisfy the estimate

$$
\left\|\left(\partial_{j} V\right) u\right\|_{q} \leq C\|u\|_{W_{q}^{2}}+C\|V u\|_{q}
$$

for all $u \in W_{q}^{2}$ with $V u \in L^{q}$.

(V2) There exists a nonnegative function $\varphi \in C^{1}\left(\mathbb{R}^{n} \backslash\{0\}, \mathbb{R}\right)$ such that

$$
4(1-\varepsilon)^{2} V \geq|\nabla \varphi|^{2} \quad \text { on } \quad \mathbb{R}^{n} \backslash\{0\} .
$$

$$
\varepsilon \liminf _{|x| \rightarrow \infty} V(x)+\omega>0 .
$$

A basic regularity result is given by:

Theorem 1. Let $V$ satisfy $(V 1)$. Let $u \in \Sigma$ solve (1.1) with $1<p<2^{*}-1$. Then:

(1) $u \in \bigcap_{2 \leq q<\infty} W_{q}^{2} \cap C^{1}$.

(2) For any multi-index $\alpha$ with $|\alpha| \leq 1$,

$$
\partial^{\alpha} u(x) \rightarrow 0 \quad \text { as } \quad|x| \rightarrow \infty .
$$

(3) If in addition $V$ satisfies $(V 1)^{\prime}$, then $u \in C^{2}$ and for any multi-index $\alpha$ with $|\alpha|=2$,

$$
\partial^{\alpha} u(x) \rightarrow 0 \quad \text { as } \quad|x| \rightarrow \infty .
$$

Remark 1.1. Assumption (V1) ensures the existence of bounded inverse $(H+$ $1)^{-1}$ in $L^{q}$ for any $q$ with $1<q<\infty$. By the definition of $H$ in $L^{q}, D(H)=$ $D(-\Delta) \cap D(V)$, where $D(-\Delta)=W_{q}^{2}$ and $D(V)=\left\{u \in L^{q} ; V u \in L^{q}\right\}$. Since $H$ and $-\Delta$ are closed in $L^{q}$ with $D(H) \subset D(-\Delta)$, it follows from the closed graph theorem that there exists a constant $C$ such that

$$
\|\Delta u\|_{q} \leq C\|(H+1) u\|_{q}
$$

for all $u \in D(H)$. Here $C$ may depend on $q$. A sufficient condition for (V1) is given by

$$
|\nabla V| \leq a V+b
$$

with $a, b \geq 0$ [24]. For $V(x)=|x|^{\theta}$ with $\theta>0$, the condition holds if $\theta \geq 1$. Another sufficient condition for (V1) is given by $V \in B_{\infty}$ for $n \geq 3$ [17, 32], namely, $V \in L_{\text {loc }}^{\infty}$ satisfies the estimate

$$
\|V\|_{L^{\infty}(B)} \leq \frac{C}{|B|} \int_{B}|V|
$$

for every ball $B \subset \mathbb{R}^{n}$. Therefore $V(x)=|x|^{\theta}$ with $\theta \geq 0$ satisfies (V1) for $n \geq 3$. 
Remark 1.2. (V1) is satisfied for $V(x)=|x|^{\theta}$ with $\theta>0$. Indeed, if $\theta \geq 1$, we use the inequality

$$
\left\||x|^{\theta-1} u\right\|_{q} \leq\left\||x|^{\theta} u\right\|_{q}^{1-1 / \theta}\|u\|_{q}^{1 / \theta}
$$

and if $\theta<1$, we use the inequality

$$
\left\||x|^{\theta-1} u\right\|_{q} \leq C\left\|(-\Delta)^{(1-\theta) / 2} u\right\|_{q} \leq C\|\Delta u\|_{q}^{(1-\theta) / 2}\|u\|_{q}^{(1+\theta) / 2},
$$

where $n<q<n /(1-\theta)$, see [10]. The condition $q>n$ in $(\mathrm{V} 1)^{\prime}$ is required for the control of $L^{\infty}$ norm by means of first derivatives in the Sobolev embedding.

Exponential decay estimates on the basis of $L^{2}, L^{p+1}, H^{1}$ spaces are given by:

Theorem 2. Let $V$ satisfy $(V 1),(V 2),(V 3)$ and let $u$ and $p$ as in Theorem 1. Then, $e^{\varphi}\left(|\nabla u|^{2}+V|u|^{2}+|u|^{2}+|u|^{p+1}\right) \in L^{1}$ and $u$ satisfies the inequalities

$$
\begin{aligned}
& \varepsilon \int e^{\varphi}\left(|\nabla u|^{2}+V|u|^{2}\right)+\omega \int e^{\varphi}|u|^{2} \\
& \leq \int e^{\varphi}|u|^{p+1} \\
& \leq(2-\varepsilon) \int e^{\varphi}\left(|\nabla u|^{2}+V|u|^{2}\right)+\omega \int e^{\varphi}|u|^{2} .
\end{aligned}
$$

As a corollary, we have local exponential decay estimates:

Corollary 1.1. Under the same assumptions as in Theorem 2,

$$
\lim _{R \rightarrow \infty} e^{\varphi_{*}(R)} \int_{R<|x|<R+1}|u|^{2}=0,
$$

where $\varphi_{*}(R)=\inf _{|x|>R} \varphi(x)$.

Remark 1.3. In the case where $V$ is radial and continuous, $\varphi$ is given by

$$
\varphi(x)=2(1-\varepsilon) \int_{0}^{|x|} \tilde{V}(\rho)^{1 / 2} d \rho,
$$

where $\tilde{V}$ is a continuous, nonnegative function on $[0, \infty)$ satisfying $\tilde{V}(|x|)=V(x)$ for all $x \in \mathbb{R}^{n}$. With this choice the inequality is realized as the equality $|\nabla \varphi|^{2}=$ $4(1-\varepsilon)^{2} V$. In particular, for $V(x)=|x|^{\theta}$ with $\theta \geq 0, \varphi$ is given by $\varphi(x)=$ $(4(1-\varepsilon) /(2+\theta))|x|^{1+\theta / 2}$.

Remark 1.4. (V3) is redundant if $\liminf _{|x| \rightarrow \infty} V(x)=\infty$ or $\omega>0$. In the case $V=0,(\mathrm{~V} 3)$ reduces to the same assumption $\omega>0$ as (8.1.5) in [3] that excludes the nonexistence of nontrivial solutions. 
Remark 1.5. For $V(x)=|x|^{\theta}$ with $\theta \geq 1$ or with $\theta \geq 0$ for $n \geq 3$, the corollary above implies that

$$
\lim _{R \rightarrow \infty} \exp \left(\frac{4(1-\varepsilon)}{2+\theta} R^{1+\theta / 2}\right) \int_{R<|x|<R+1}|u|^{2}=0,
$$

which is optimal for $\theta>0$ in the sense that for any $\varepsilon>0$

$$
\lim _{R \rightarrow \infty} \exp \left(\frac{4(1+\varepsilon)}{2+\theta} R^{1+\theta / 2}\right) \int_{R<|x|<R+1}|u|^{2}=\infty .
$$

See Example 5.1 of Uchiyama [37], where we apply $q_{1}(x)=|x|^{\theta}+\omega, q_{2}(x)=$ $-|u|^{p-1}, \beta=(2+\theta) / 2, \mu^{*}=2 /(2+\theta)$ in the notation of [37]. We note that $q_{2}(x)=o(1)$ as $|x| \rightarrow \infty$ by Theorem 1 .

For pointwise exponential decay estimates, we introduce an assumption on the potential in terms of $\varphi$ in (V2).

(V4) For any $\delta, a>0$ there exists $C_{\delta}>0$ such that

$$
\varphi(x) \leq(1+\delta) \varphi(y)+C_{\delta}
$$

for any $x \in \mathbb{R}^{n}$ and all $y \in B(x ; a) \equiv\{y ;|x-y| \leq a\}$.

The assumption (V4) regulates a local growth behavior by means of the uniform rate $1+\delta$. In the case $V(x)=|x|^{\theta}$ with $\theta \geq 0$, (V4) is satisfied.

Theorem 3. Let $V$ satisfy $(V 1),(V 2),(V 3),(V 4)$. Let $u \in \Sigma$ solve (1.1) with $1<p<2^{*}-1$. Then for any $\delta>0$

$$
\sup _{x \in \mathbb{R}^{n}} \exp (\varphi(x) /(1+\delta)(n+2))|u(x)|<\infty .
$$

Remark 1.6. For $V=0$, Theorem 3 reduces to Theorem 8.1.1 in Cazenave [3]. As for the pointwise decay in the case where $V(x)=|x|^{2}$, we refer the reader to $[11,12]$.

We prove Theorems 1, 2, and 3 in Sections 2, 3, and 4, respectively. Although the method of proof below is standard, several improvements are made to take the effect of potentials into account (compare with [3]). For completeness, we repeat some of the standard arguments besides new ingredients. We finally note that all the statements above and the arguments below are still valid for more general nonlinearities of the form $\lambda|u|^{p-1} u$ with $\lambda \in \mathbb{C}$, and that the method in this paper provides an improvement of the result by Kurth [18] which showed the exponentially fast decay of nonlinear modes for a nonlocal nonlinear Schrödinger equation related to dispersion-managed solutions. 


\section{Proof of Theorem 1}

Let $u \in \Sigma$ solve (1.1). We write the equation in $\Sigma^{\prime}$ as

$$
(H+1) u=(1-\omega) u+|u|^{p-1} u .
$$

By the Sobolev embedding, $u \in L^{p+1}$ and therefore $|u|^{p-1} u \in L^{(p+1) / p}$. We rewrite (2.1) as

$$
u=(1-\omega)(H+1)^{-1} u+(H+1)^{-1}|u|^{p-1} u,
$$

where, by $(V 1)$ the first and second terms on the RHS make sense in $L^{p+1} \cap L^{2}$ and in $L^{(p+1) / p}$, respectively.

For $j \geq 1$, we define $r_{j}, q_{j}$ as

$$
\frac{1}{r_{j}}=\frac{1}{p+1}-\frac{2 j}{n}, \frac{1}{q_{j}}=-\delta p^{j}+\frac{2}{n(p-1)},
$$

where

$$
\delta=\frac{2}{n(p-1)}-\frac{1}{p+1}
$$

We note here that $\delta>0,1 / r_{j+1}=1 / r_{j}-2 / n, 1 / q_{j+1}=p / q_{j}-2 / n, 1 / q_{j}>1 / r_{j}$ and that $1 / q_{j}$ and $1 / r_{j}$ are decreasing and tend to $-\infty$ as $j \rightarrow \infty$.

We claim that $u \in L^{r_{j}}+L^{q_{j}}$ for any $j$ with $1 / r_{j}, 1 / q_{j}>0$. By the Sobolev embedding and Remark 1.1, we have

$$
(H+1)^{-1} u \in L^{r} \quad \text { and } \quad(H+1)^{-1}|u|^{p-1} u \in L^{q}
$$

for any $r$ and $q$ with $1 /(p+1) \geq 1 / r \geq 1 /(p+1)-2 / n$ and $p /(p+1) \geq 1 / q \geq$ $p /(p+1)-2 / n$. The claim for $j=1$ follows from (2.2). We assume that $u \in$ $L^{r_{j-1}}+L^{q_{j-1}}$ for some $j \geq 2$ and prove that $u \in L^{r_{j}}+L^{q_{j}}$. By assumption, the Sobolev embedding and Remark 1.1, we have

$$
(H+1)^{-1} u \in L^{r}+L^{\tilde{r}}
$$

for any $r$ and $\tilde{r}$ with $1 / r_{j-1} \geq 1 / r \geq 1 / r_{j-1}-2 / n$ and $1 / q_{j-1} \geq 1 / \tilde{r} \geq 1 / q_{j-1}-2 / n$. Then we may choose $r=r_{j}$ and $\tilde{r}=q_{j}$ since $1 / q_{j-1}>1 / q_{j}>1 / q_{j-1}-2 / n$. Since $|u|^{p-1} u \in L^{r_{j-1} / p}+L^{q_{j-1} / p}$, in the same way as above,

$$
(H+1)^{-1}|u|^{p-1} u \in L^{q}+L^{\tilde{q}}
$$

for any $q$ and $\tilde{q}$ with $p / r_{j-1} \geq 1 / q \geq p / r_{j-1}-2 / n$ and $p / q_{j-1} \geq 1 / \tilde{q} \geq p / q_{j-1}-$ $2 / n$. Then we may choose $q$ and $\tilde{q}$ as $1 / q=p / r_{j-1}-2 / n$ and $1 / \tilde{q}=p / q_{j-1}-2 / n=$ $1 / q_{j}$ so that $1 / q_{j}=1 / \tilde{q}>1 / q>1 / r_{j}$. The claim for $j$ follows from (2.2).

Repeating a similar argument, we see that

$$
u \in L^{r}+L^{q}
$$


for any $r$ and $q$ sufficiently large. This implies that

$$
u \in \bigcap_{2 \leq q<\infty} L^{q} .
$$

Part (1) then follows from (2.2), Remark 1.1, and the Sobolev embedding $W_{q}^{2} \subset C^{1}$ for $q>n$. Moreover, $\nabla u \in L^{\infty}$ and therefore $u$ is globally Lipschitz continuous. Since $u \in H^{1}$, Part (2) holds. For Part (3), we take the distributional derivatives of (1.1) to have

$$
(H+1) \partial_{j} u=-\left(\partial_{j} V\right) u+(1-\omega) \partial_{j} u+\partial_{j}\left(|u|^{p-1} u\right) .
$$

By Part (1), the terms on the RHS of (2.3) belong to $L^{q}$, where $q>n$ is as in (V1)'. Therefore, by (2.3) and Remark 1.1, we have $\partial_{j} u \in W_{q}^{2} \subset C^{1,1-n / q}$. This proves Part (3).

\section{Proof of Theorem 2}

Let $\zeta=\exp \varphi$ and let $\zeta_{m}=\exp (m \varphi /(m+\varphi))$ for $m \geq 1$. Taking the real part of the scalar product of (1.1) with $\zeta_{m} u$, we obtain

$$
\operatorname{Re} \int \nabla\left(\zeta_{m} u\right) \cdot \nabla \bar{u}+\int \zeta_{m} V|u|^{2}+\omega \int \zeta_{m}|u|^{2}=\int \zeta_{m}|u|^{p+1} .
$$

The first term on the RHS of (3.1) is equal to

$$
\int \zeta_{m}|\nabla u|^{2}+\operatorname{Re} \int \zeta_{m} \frac{m^{2} \nabla \varphi}{(m+\varphi)^{2}} \cdot u \nabla \bar{u}
$$

where the second term is estimated as

$$
\begin{aligned}
\left|\operatorname{Re} \int \zeta_{m} \frac{m^{2} \nabla \varphi}{(m+\varphi)^{2}} \cdot u \nabla \bar{u}\right| & \leq \int \zeta_{m}|\nabla \varphi \| u||\nabla u| \\
& \leq(1-\varepsilon) \int \zeta_{m}|\nabla u|^{2}+\frac{1}{4(1-\varepsilon)} \int \zeta_{m}|\nabla \varphi|^{2}|u|^{2} .
\end{aligned}
$$

Combining these estimates with (3.1) and applying (V2), we obtain

$$
\begin{aligned}
& \varepsilon \int \zeta_{m}\left(|\nabla u|^{2}+V|u|^{2}\right)+\omega \int \zeta_{m}|u|^{2} \\
& \leq \int \zeta_{m}|u|^{p+1} \\
& \leq(2-\varepsilon) \int \zeta_{m}\left(|\nabla u|^{2}+V|u|^{2}\right)+\omega \int \zeta_{m}|u|^{2} .
\end{aligned}
$$

We now distinguish between two cases : (1) $\omega>0$. (2) $\omega \leq 0$.

(1) The case $\omega>0$. By Theorem 1 (2), there exists $R>0$ such that 
$|u(x)|^{p-1} \leq \omega / 2$ for all $x$ with $|x| \geq R$. By (3.2) we obtain

$$
\varepsilon \int \zeta_{m}\left(|\nabla u|^{2}+V|u|^{2}\right)+\frac{\omega}{2} \int \zeta_{m}|u|^{2} \leq \int_{|x| \leq R} \zeta|u|^{p+1}
$$

where we have used the inequality $\zeta_{m} \leq \zeta$. Since the RHS of (3.3) is finite and independent of $m$, Fatou's lemma implies that $\zeta\left(|\nabla u|^{2}+V|u|^{2}+|u|^{2}\right) \in L^{1}$ and

$$
\varepsilon \int \zeta\left(|\nabla u|^{2}+V|u|^{2}\right)+\frac{\omega}{2} \int \zeta|u|^{2} \leq \int_{|x| \leq R} \zeta|u|^{p+1}
$$

which together with (3.2) in turn implies that $\zeta|u|^{p+1} \in L^{1}$. By the monotone convergence theorem, (3.2) holds with $\zeta_{m}$ replaced by $\zeta$.

(2) The case $\omega \leq 0$. By (V3), there exist $\eta, R^{\prime}>0$ such that $\varepsilon V(x)+\omega \geq \eta$ for all $x$ with $|x| \geq R^{\prime}$. By Theorem 1 (2), there exists $R>0$ such that $|u(x)|^{p-1} \leq \eta / 2$ for all $x$ with $|x| \geq R$. We estimate the quadratic part with respect to $u$ in (3.2) from below as

$$
\begin{aligned}
& \varepsilon \int \zeta_{m} V|u|^{2}+\omega \int \zeta_{m}|u|^{2} \\
& =\left(\int_{|x| \leq R^{\prime}}+\int_{|x|>R^{\prime}}\right)(\varepsilon V+\omega) \zeta_{m}|u|^{2} \\
& \geq \int_{|x| \leq R^{\prime}}(\varepsilon V+\omega) \zeta_{m}|u|^{2}+\eta \int_{|x|>R^{\prime}} \zeta_{m}|u|^{2} .
\end{aligned}
$$

The middle term in (3.2) is bounded as

$$
\int \zeta_{m}|u|^{p+1} \leq \int_{|x| \leq R} \zeta_{m}|u|^{p+1}+\frac{\eta}{2} \int_{|x|>R} \zeta_{m}|u|^{2} .
$$

By (3.2), (3.4) and (3.5) we obtain

$$
\begin{aligned}
& \varepsilon \int \zeta_{m}|\nabla u|^{2}+\eta \int_{|x|>R^{\prime}} \zeta_{m}|u|^{2}-\frac{\eta}{2} \int_{|x|>R} \zeta_{m}|u|^{2} \\
& \leq \int_{|x| \leq R} \zeta_{m}|u|^{p+1}-\int_{|x| \leq R^{\prime}}(\varepsilon V+\omega) \zeta_{m}|u|^{2} .
\end{aligned}
$$


If $R^{\prime}>R$, then (3.6) yields

$$
\begin{aligned}
& \varepsilon \int \zeta_{m}|\nabla u|^{2}+\frac{\eta}{2} \int_{|x|>R^{\prime}} \zeta_{m}|u|^{2} \\
& \leq \frac{\eta}{2} \int_{R<|x| \leq R^{\prime}} \zeta_{m}|u|^{2}+\int_{|x| \leq R} \zeta_{m}|u|^{p+1}-\int_{|x| \leq R^{\prime}}(\varepsilon V+\omega) \zeta_{m}|u|^{2} .
\end{aligned}
$$

If $R \geq R^{\prime}$, then (3.6) yields

$$
\begin{aligned}
& \varepsilon \int \zeta_{m}|\nabla u|^{2}+\frac{\eta}{2} \int_{|x|>R} \zeta_{m}|u|^{2} \\
& \leq-\eta \int_{R^{\prime}<|x| \leq R} \zeta_{m}|u|^{2}+\int_{|x| \leq R} \zeta_{m}|u|^{p+1}-\int_{|x| \leq R^{\prime}}(\varepsilon V+\omega) \zeta_{m}|u|^{2} .
\end{aligned}
$$

Taking both (3.7) and (3.8) into account, we have

$$
\varepsilon \int \zeta_{m}|\nabla u|^{2}+\frac{\eta}{2} \int \zeta_{m}|u|^{2} \leq\left(\frac{\eta}{2}-\omega\right) \int_{|x| \leq \max \left(R, R^{\prime}\right)} \zeta|u|^{2}+\int_{|x| \leq R} \zeta|u|^{p+1} .
$$

By Fatou's lemma, $\zeta\left(|\nabla u|^{2}+|u|^{2}\right) \in L^{1}$. By the first inequality in (3.2),

$$
\begin{aligned}
& \varepsilon \int \zeta_{m} V|u|^{2} \\
& \leq-\omega \int \zeta_{m}|u|^{2}+\int \zeta_{m}|u|^{p+1} \\
& \leq\left(\frac{\eta}{2}-\omega\right) \int \zeta|u|^{2}+\int_{|x| \leq R} \zeta|u|^{p+1}
\end{aligned}
$$

Similarly, we have $\zeta V|u|^{2} \in L^{1}$. By the second inequality in (3.2) we have $\zeta|u|^{p+1} \in$ $L^{1}$ since, by the monotone convergence theorem, the RHS of the second inequality in (3.2) holds with $\zeta_{m}$ replaced by $\zeta$. This proves Theorem 2 .

\section{Proof of Theorem 3}

By the Sobolev embedding $W_{q}^{1} \hookrightarrow L^{\infty}$ for $q>n$ and Theorem $1, \nabla u \in L^{\infty}$. Let $L=\|\nabla u\|_{\infty}+\eta$ with $\eta>0$. For any $x \in \mathbb{R}^{n}$ we define

$$
B(x)=B(x ;|u(x)| / 2 L)=\left\{y \in \mathbb{R}^{n} ;|x-y| \leq|u(x)| / 2 L\right\} .
$$

For any $y \in B(x)$, we obtain

$$
\begin{aligned}
|u(x)|^{2} & \leq(|u(y)|+L|x-y|)^{2} \\
& \leq 2|u(y)|^{2}+2 L^{2}|x-y|^{2} \\
& \leq 2|u(y)|^{2}+\frac{1}{2}|u(x)|^{2}
\end{aligned}
$$


and therefore

$$
|u(x)|^{2} \leq 4|u(y)|^{2} .
$$

Integrating the above inequality with respect to $y$ over $B(x)$, we have

$$
C_{n}\left(\frac{|u(x)|}{2 L}\right)^{n}|u(x)|^{2} \leq 4 \int_{B(x)}|u(y)|^{2} d y .
$$

This implies, with $a=\|u\|_{\infty} / 2 L$,

$$
\begin{aligned}
\exp \left(\frac{\varphi(x)}{1+\delta}\right)|u(x)|^{2+n} & \leq \frac{4(2 L)^{n}}{C_{n}} \exp \left(\frac{C_{\delta}}{1+\delta}\right) \int_{B(x)} \exp (\varphi(y))|u(y)|^{2} d y \\
& \leq \frac{4(2 L)^{n}}{C_{n}} \exp \left(\frac{C_{\delta}}{1+\delta}\right) \int e^{\varphi}|u|^{2},
\end{aligned}
$$

as was to be shown.

\section{Discussion}

As we mentioned in the end of Introduction, the method above provides the Gaussian decay for the solutions of

$$
-u_{x x}+x^{2} u+\omega u-F(u)=0, \quad x \in \mathbb{R}, \quad \omega \in \mathbb{R},
$$

where

$$
F(u):=\int_{0}^{1} S^{-1}(z)\left(\frac{1}{T(z)}|S(z) u|^{2} S(z) u\right) d z,
$$

with $S(z):=U\left(R^{\mathrm{eff}}(z)\right)$. Here, $U(z)$ denotes the group generated by the harmonic oscillator, $T(z)$ is a characteristic pulse width and $R^{\text {eff }}(z)$ is the effective residual dispersion (for details, see [18]). This problem is related to dispersion managed solitons (see, e.g., $[18,28,35]$ ). Our method, particularly the way of proof of Theorem 2 is applicable to the equation (5.1) since the effect of coefficient to the quadratic factor in the functional associated with the nonlocal nonlinearity $F(u)$ is small far away from the origin as in the case of $|u|^{p-1} u$. This improves the result in [18] concerning the exponential decay of solutions of (5.1).

\section{Acknowledgement}

This work started while one of us (R. F.) stayed at the Université de Paris-Sud, Orsay. R. F. acknowledges the warm hospitality of Professors Jean-Claude Saut and Anne de Bouard. 


\section{References}

[1] H. Berestycki and T. Cazenave, Instabilité des états stationnaires dans les équations de Schrödinger et de Klein-Gordon non linéaires, C. R. Acad. Sci. Paris 293 (1981), 489-492.

[2] H. Berestycki and P. L. Lions, Nonlinear scalar field equations, I-Existence of a ground state, Arch. Rat. Mech. Anal. 82 (1983), 313-346.

[3] T. Cazenave, Semilinear Schrödinger equations, Courant Lecture Notes in Mathematics 10, New York University, New York 2003.

[4] T. Cazenave and P. L. Lions, Orbital stability of standing waves for some nonlinear Schrödinger equations, Commun. Math. Phys. 85 (1982) 549-561.

[5] R. Fukuizumi, Stability and instability of standing waves for the nonlinear Schrödinger equation with harmonic potential, Discrete Contin. Dynam. Systems 7 (2001), 525-544.

[6] R. Fukuizumi, Stability of standing waves for nonlinear Schrödinger equations with critical power nonlinearity and potentials, Adv. Differential Equations 10 (2005), 259-276.

[7] R. Fukuizumi and M. Ohta, Instability of standing waves for nonlinear Schrödinger equations with potentials, Differential and Integral Eqs. 16 (2003), 691-706.

[8] R. Fukuizumi and M. Ohta, Stability of standing waves for nonlinear Schrödinger equations with potentials, Differential and Integral Eqs. 16 (2003), 111-128.

[9] M. Grillakis, J. Shatah and W. Strauss, Stability theory of solitary waves in the presence of symmetry I, J. Funct. Anal. 74 (1987), 160-197.

[10] I. W. Herbst, Spectral theory of the operator $\left(p^{2}+m^{2}\right)^{1 / 2}-Z e^{2} / r$, Commun. Math. Phys. 53 (1977), 285-294.

[11] M. Hirose and M. Ohta, Structure of positive radial solutions to scalar field equations with harmonic potential, J. Differential Eqs. 178 (2002), 519-540

[12] M. Hirose and M. Ohta, Uniqueness of positive solutions to scalar field equations with harmonic potential, Preprint.

13] T. Kato, Schrödinger operators, in Nonlinear Schrödinger equations, 218-263, Lecture Notes in Phys., 345, Springer, Berlin 1989.

[14] Y. Kabeya and K. Tanaka, Uniqueness of positive radial solutions of semilinear elliptic equations in $R^{N}$ and Séré's non-degeneracy condition, Commun. Partial Diff. Eq. 24 (1999), $563-598$.

[15] O. Kavian and F. Weissler, Self similar solutions of the pseudo-conformally invariant nonlinear Schrödinger equation, Michigan. Math. J. 41 (1994), 151-173.

[16] M. Kunze, T. Küpper, V. K. Mezentsev, E.G. Shapiro, S. K. Turitsyn, Nonlinear solitary waves with Gaussian tails, Physica D 128 (1999), 273-295.

[17] K. Kurata and S. Sugano, A remark on estimates for uniformly elliptic operators on weighted $L^{p}$ spaces and Morrey spaces, Math. Nachr. 209 (2000), 137-150.

[18] M. Kurth, On the existence of infinitely many modes of a nonlocal NLS related to DM solitons, Siam J. Math. Anal. 36 (2004), 967-985.

[19] M. K. Kwong, Uniqueness of positive solutions of $\Delta u-u+u^{p}=0$ in $\mathbb{R}^{n}$, Arch. Rational. Mech. Anal. 105 (1989), 234-266.

[20] P. L. Lions, The concentration-compactness principle in the calculus of variations, the locally compact case, part I, Ann. Inst. H. Poincaré. Anal. Nonlinéaire 1 (1984), 109-145.

[21] P. L. Lions, The concentration-compactness principle in the calculus of variations, the locally compact case, part II, Ann. Inst. H. Poincaré. Anal. Nonlinéaire 1 (1984), 223-282.

[22] Y. G. Oh, Cauchy problem and Ehrenfest's law of nonlinear Schrödinger equations with potentials, J. Differential Eqs. 81 (1989), 255-274.

[23] Y. G. Oh, Stability of semiclassical bound states of nonlinear Schrödinger equations with potentials, Commun. Math. Phys. 121 (1989), 11-33.

[24] N. Okazawa, An $L^{p}$ theory for Schrödinger operators with nonnegative potentials, J. Math. Soc. Japan. 36 (1984), 675-688.

[25] N. Okazawa, $L^{p}$-theory of Schrödinger operators with strongly singular potentials, Japan. J. Math. 22 (1996), 199-239. 
[26] P. H. Rabinowitz, On a class of nonlinear Schrödinger equations, Z. Angew. Math. Phys. 43 (1992), 270-291.

[27] H. A. Rose and M. I. Weinstein, On the bound states of the nonlinear Schrödinger equation with a linear potential, Physica D 30 (1988), 207-218.

[28] T. Schäfer, V. K. Mezentsev, K. H. Spatschek and S. K. Turitsyn, The dispersion-managed soliton as a ground state of a macroscopic nonlinear quantum oscillator, Proceedings of the Royal Society London A 457 (2001), 273-282.

[29] Y. A. Semenov, Schrödinger operators with $L_{\text {loc }}^{q}$-potentials, Commun. Math. Phys. 53 (1977), 277-284.

[30] J. Shatah, Stable standing waves for nonlinear Klein-Gordon equations, Commun. Math. Phys. 91 (1983), 313-327.

[31] J. Shatah and W. Strauss, Instability of nonlinear bound states, Commun. Math. Phys. 100 (1985), 173-190.

[32] Z. Shen, $L^{p}$ estimates for Schrödinger operators with certain potentials, Ann. Inst. Fourier, Grenoble 45 (1995), 513-546.

[33] W. Strauss, Existence of solitary waves in higher dimensions, Commun. Math. Phys. 55 (1977), 149-162.

[34] C. Sulem and P.-L. Sulem, The nonlinear Schrödinger equation. Self-focusing and wave collapse, Applied Mathematical Sciences, 139. Springer-Verlag, New York 1999.

[35] S. K. Turitsyn, T. Schäfer, K. H. Spatschek and V. K. Mezentsev, Path-averaged chirped optical soliton in dispersion-managed fiber communication lines, Optical Communications 163 (1999), 122-158.

[36] J. Uchiyama, Lower bounds of decay order of eigenfunctions of second-order elliptic operators, Publ. Res. Inst. Math. Sci. 21 (1985), 1281-1297.

[37] J. Uchiyama, Decay order of eigenfunctions of second-order elliptic operators in an unbounded domain and its applications, Publ. Res. Inst. Math. Sci. 22 (1986), 1079-1104.

[38] M. I. Weinstein, Nonlinear Schrödinger equations and sharp interpolation estimates, Commun. Math. Phys. 87 (1983), 567-576.

[39] M. I. Weinstein, Lyapunov stability of ground states of nonlinear dispersive evolution equations, Comm. Pure Appl. Math. 39 (1986), 51-68.

[40] J. Zhang, Stability of standing waves for the nonlinear Schrödinger equations with unbounded potentials, Z. Angew. Math. Phys. 51 (2000), 489-503.

[41] J. Zhang, Stability of Attractive Bose-Einstein Condensates, Journal of Statistical Physics 101 (2000), 731-745.

Reika Fukuizumi and Tohru Ozawa

Department of Mathematics

Hokkaido University

Sapporo 060-0810

Japan

(Received: May 4, 2004)

\section{To access this journal online: \\ (4) http://www.birkhauser.ch}

